\title{
Parasitism drives hostoffspring microbiome with significant influence in parasite epidemic
}

\author{
Xueke Gao ${ }^{1}$, Junyu Luo ${ }^{1}$, Jichao $\mathrm{Ji}^{1}$, Lin Niu${ }^{1}$, Xiangzhen Zhu ${ }^{1}$, Li Wang ${ }^{1}$, Shuai Zhang ${ }^{1}$, \\ and Jinjie Cui ${ }^{1}$ \\ ${ }^{1}$ Chinese Academy of Agricultural Sciences Cotton Research Institute
}

July 8, 2020

\begin{abstract}
The relationship of host and symbionts is complex and dynamic. Symbionts can significantly impact host phenotypes and parasite epidemics may be influenced by interactions among symbionts. Aphids are well known for their symbiotic associations with bacteria. However, few studies have examined the offsprings of parasitized host and the ecological implications of a dynamic microbiome longitudinaly. In the present study, we surveyed the microbiota in non-parasitized aphids and parasitized aphids its offspring for over four consecutive generations by using high-throughput $16 \mathrm{~S}$ rRNA sequencing. Across hosts, parasite strongly altered symbiont composition of parasitized aphids offspring, especially in the fourth generation. Moreover, parasitism reduced weight and reproductive capacity of the parasitized offspring and influenced parasite epidemics. Taken together, these results indicate that parasitoids can influence host-microbiome interactions by altering the symbionts composition in the host offspring. Our findings further supports the importance host-parasite-microbiome tirad interactions, which can create intense reciprocal selection resulting in coevolution between species.
\end{abstract}

\section{Hosted file}

Manuscript.docx available at https://authorea.com/users/340966/articles/468038-parasitismdrives-hostoffspring-microbiome-with-significant-influence-in-parasite-epidemic 